\title{
Capillary wave spectrum at adsorbed liquid films
}

\author{
Eva M. Fernández* \\ Instituto de Ciencia de Materiales de Madrid, CSIC, 28049 Madrid, Spain
}

Enrique Chacón ${ }^{\dagger}$

Instituto de Ciencia de Materiales de Madrid, CSIC, 28049 Madrid, and Instituto de Ciencia de Materiales Nicolás Cabrera, Universidad Autońma de Madrid, 28049 Madrid, Spain

Pedro Tarazona

Departamento de Física Teórica de la Materia Condensada, Universidad Autónoma de Madrid, Madrid 28049, and Instituto de Ciencia de Materiales Nicolás Cabrera, Universidad Autónoma de Madrid, 28049 Madrid, Spain

(Received 28 February 2012; revised manuscript received 24 April 2012; published 1 August 2012)

\begin{abstract}
Capillary wave fluctuations at the edges of liquid wetting layers are analyzed in Monte Carlo simulations of a Lennard-Jones fluid adsorbed on a planar wall substrate. The analysis is based on the Fourier modes of the liquid surface, constructed via the intrinsic sampling method. For films thinner than four molecular layers we can quantify the damping of the capillary waves due to the wall potential. Our results are presented in the theoretical framework of the effective surface Hamiltonians, to establish a quantitative link between the molecular structure and the mesoscopic descriptions used for renormalization-group analysis. We observe the predicted exponential decay of the wall damping, with the correlation length of the liquid bulk. However, associated with the molecular layering of the density profiles, we observe a strong oscillatory dependence with respect to the mean film thickness, not included in any theoretical prediction. We discuss the possibility of finite-size effects in this respect. Three different definitions of the intrinsic surface at molecular level are tested, to show that the square gradient terms of the Hamiltonian are robust, while the surface bending (or nonlocal) terms are tied to each specific definition.
\end{abstract}

DOI: 10.1103/PhysRevB.86.085401

PACS number(s): 68.08.Bc, 68.03.Kn, 68.03.Hj

\section{INTRODUCTION}

Interfacial phenomena are important in nature and they have a great number of practical applications, e.g., the structure of the lipid bilayers that form cell membranes, the stability of colloids in emulsions (such as milk and paint), etc. At mesoscopic scale, these systems are usually studied through an effective Hamiltonian in terms of the position and shape of the interface. For liquid films adsorbed on solid substrates the local thickness of the film $\xi(\mathbf{R})$ is the relevant collective variable. ${ }^{1}$ The simplest Hamiltonian models have two separated contributions: the first one is given by the surface tension of the free liquid-vapor interface, $\gamma_{0}$, multiplied by the area of the corrugated surface; and the second contribution represents the interaction of the adsorbed film with the substrate through an effective potential $\Phi(\xi)$, which, in first approximation, is considered local. In general the predictions of these local Hamiltonians are very accurate, although the renormalization-group ( $R G$ ) analysis is not supported by the experimental and simulation results. ${ }^{2,3}$ Two nonlocal effective Hamiltonians have been proposed in this context: the first one introduces the nonlocal effects in the interfacial tension, ${ }^{3}$ by means of a surface tension that depends on the film thickness. In the second proposal the nonlocal effects are included in the interaction between the substrate and the liquidvapor interface, i.e., in the effective interface potential. ${ }^{4,5}$ In the latter model the nonlocal effects are due to a self-interaction arising from two-body interfacial forces between particles on the interface and also are presented for free liquid-vapor interfaces. ${ }^{6}$ Although these Hamiltonians have been broadly used in the study of the criticality for short-range wetting transitions, the quantitative identification of these nonlocal effects in realistic models of wetting films has proven to be difficult. This is the aim of the present work: to study the dependence of surface fluctuations on the thickness of the adsorbed liquid film and to analyze possible nonlocal effects. This is a very delicate question because previous studies ${ }^{4,5}$ have confirmed the validity of the simplest mean-field (MF)like Hamiltonian until the film layer is rather thin, and then the theoretical assumptions, based on a smooth density profile at the external edge of the film, may be questioned.

The experimental observation of nonlocal effects on the fluctuations of wetting layers is very difficult. X-ray diffuse scattering shows that the surface structure of absorbed films is well characterized by the undulations due to the roughness of the solid and the thermal capillary waves (CWs) of the film, ${ }^{7}$ but the data lack the accuracy to discriminate the effects beyond a simple local Hamiltonian, without any $\mathrm{CW}$ damping from the wall. A promising alternative is to use computer simulations, which may provide full information on the structures at the molecular level. Recently, Pang et al. ${ }^{8}$ analyzed the correlation functions of large-scale Monte Carlo (MC) simulations of interfaces in the Ising model to present, for the first time, some evidence in favor of the nonlocal interface Hamiltonian. However, despite the large system sizes used in that study, the conclusions were not fully clear and more tests are necessary. Our aim is to study the nonlocal effects in a more realistic system like a Lennard-Jones (LJ) fluid, which could be used to predict the quantitative contribution of these effects in experiments, and, at the same time, to avoid the usual path in which the fluctuations of the liquid surface are inferred 
from density-density correlations near the liquid surface. Over the last decade, the study of CW fluctuations at the free liquid surface has taught us to be very cautious about this approach. The density profiles and the two-particle density correlations contain a limited amount of information on the structure of the liquid surface, and they unavoidably mix the averages on the instantaneous shape of the interface with the density fluctuations near the interface. In the long-wavelength limit, the divergence of $\mathrm{CW}$ fluctuations leaves a clear signal in the density-density fluctuations, ${ }^{9}$ but that regime is well described by the classical form of the CW theory (CWT) in terms of the macroscopic surface tension. Any deviation from that macroscopic limit has to be searched at shorter scales, for surface fluctuations that are not too far above the molecular size; but then the link between the mesoscopic description in terms of $\xi(\mathbf{R})$ and the molecular positions becomes less certain.

In our approach we set a definition for $\xi(\mathbf{R})$ directly in terms of the molecular positions, so that the statistical sampling, and the analysis of the mesoscopic Hamiltonian $\mathcal{H}[\xi]$, may be done from the molecular configurations along a computer simulation, without any need to rely on the density distribution or the two-particle correlations. Repeating the analysis with other reasonable definitions for $\xi(\mathbf{R})$ gives perspective to discern which properties of $\mathcal{H}[\xi]$ are generic, i.e., independent of the detailed definition for the local film thickness.

The application of this approach to the instantaneous intrinsic surface (IS), which represents the microscopic frontier between the liquid and the vapor phases, has provided a robust link between the CWT and the molecular structure of fluid interfaces. In that process we have learned that to get a good separation of the CWs from the bulk-like compressibility fluctuations, the IS must be defined as the boundary of the percolation cluster in a percolation analysis of the slab liquid. ${ }^{10}$ Several proposals with this scheme have been published over the years, ${ }^{11-17}$ and a critical revision of the different proposals was reported in two recent reviews. ${ }^{18,19}$ For the present study we have chosen the first practical scheme: the intrinsic sampling method (ISM), ${ }^{10,16,17,20}$ which is computationally more demanding than the other but is the most suitable for study of liquid-vapor surfaces. The crucial concept of the ISM is the self-consistent identification of particles belonging to the outmost layer of the liquid and the determination of the IS $\xi(\mathbf{R})$, as the minimal area surface going through that set of particles.

As the previous step to the present work, in a recent paper ${ }^{21}$ we showed that the mean position of the IS $\xi_{\text {IS }}=\langle\xi(\mathbf{R})\rangle$ given by the ISM is a very accurate way to define the thickness of an adsorbed liquid layer on a solid substrate. This film thickness definition follows with quantitative accuracy the predictions of simple local Hamiltonians, even for films as thin as one monolayer, and the effective interfacial potential $\Phi(\xi)$ has the simplest exponential form with a surprising accuracy, from a single monolayer to very thick films. In this paper we have expanded that previous work to study the effect of the substrate on the CW spectrum at the liquid-vapor interface of adsorbed liquid films of different thicknesses.

\section{MODEL}

We have studied the simple case of complete wetting on an inert structureless substrate, far from the critical region.
The adsorbed film is an LJ fluid truncated at a cutoff distance of $2.5 \sigma$ and a temperature $k_{b} T / \epsilon \equiv(\beta \epsilon)^{-1}=0.75$. We use the parameters of the LJ potential as units of energy $\epsilon$ and distance $\sigma$. The substrate acts as a planar wall with an external potential on the fluid particles $V_{\mathrm{sf}}(z)$, which depends only on the distance to the wall plane. In particular, we use for the solid-fluid interaction a sum over three layers with the density $\rho_{\text {lay }}=1.143 \sigma^{2}$ put at $z_{i}=0.0,-0.65$, and $-1.30 \sigma$, with a truncated $4-10$ potential cut at $\mathrm{r}_{c}=2.5 \sigma_{\mathrm{sf}}$ :

$$
\begin{aligned}
\frac{V_{\mathrm{sf}}(z)}{8 \pi \epsilon_{\mathrm{sf}} \sigma_{\mathrm{sf}} \rho_{\mathrm{lay}}}= & \sum_{i=1}^{3}\left\{\left[\frac{1}{10}\left(\frac{\sigma_{\mathrm{sf}}}{\left(z-z_{i}\right)}\right)^{10}-\frac{1}{4}\left(\frac{\sigma_{\mathrm{sf}}}{\left(z-z_{i}\right)}\right)^{4}\right]\right. \\
& \left.-\left[\frac{1}{10}\left(\frac{\sigma_{\mathrm{sf}}}{r_{c}}\right)^{10}-\frac{1}{4}\left(\frac{\sigma_{\mathrm{sf}}}{r_{c}}\right)^{4}\right]\right\}
\end{aligned}
$$

where $\epsilon_{\mathrm{sf}}=1.3 \epsilon$ and $\sigma_{\mathrm{sf}}=0.912 \sigma$. This model potential has been used by Velasco et al. ${ }^{22,23}$ and it gives complete wetting by the LJ liquid at the temperature used here. A purely repulsive potential is considered at $z=L_{z}$, to close the simulation cell along the $Z$ axis, with the usual periodic boundary conditions on the $X$ and $Y$ directions.

We have run series of canonical ensemble MC simulations with $N=150-2000 \mathrm{LJ}$ particles, to analyze adsorbed films up to thickness of $23.5 \sigma$, in a simulation box with $L_{x}=L_{y}=$ $10.457 \sigma$ and $L_{z}=65.36 \sigma$. The ISM was applied to get the IS associated with the external edge of the adsorbed film, for which we have averaged over $10^{4}$ configurations separated by $2000 \mathrm{MC}$ steps, after $20 \times 10^{6}$ equilibration steps. In the ISM the IS, $\xi(\mathbf{R})$, is defined by a set of molecules called surface pivots, $N_{s}$, chosen to represent the instantaneous liquid surface. The number of these pivots per unit area, $n_{s}=N_{s} / A_{0}$, is the main control parameter of the ISM. The optimal value of $n_{s}$ may be consistently determined from a wide range of structural and dynamical properties of the interface. ${ }^{16}$ In our case we have used the structural criterion and obtained an optimal occupation of $n_{s} \sigma^{2}=0.70 \pm 0.05$ for all the film thickness analyzed here, although we must note that for very thin adsorbed films the determination of $n_{s}$ becomes less robust. The IS is described through its Fourier components:

$$
\xi\left(\mathbf{R}, q_{u}\right)=\hat{\xi}_{0}+\sum_{0<|\mathbf{q}| \leqslant q_{\max }} \hat{\xi}_{q} e^{i \mathbf{q} \cdot \mathbf{R}} .
$$

From here on, we assume periodic boundary conditions on a square transverse section $A_{0}=L_{x}^{2}$ on the $X Y$ plane, parallel to the mean orientation of the interface. The wave vectors allowed by the periodic boundary conditions are $\mathbf{q}=2 \pi(\mu, \nu) / L_{x}$ with $\mu, \nu=0, \pm 1, \pm 2, \ldots$ For a noncorrugated wall the statistical averages of $\hat{\xi}_{q}$ vanish for any $q \neq 0$, while $\xi_{\text {IS }}=\langle\xi(\mathbf{R})\rangle=\left\langle\hat{\xi}_{0}\right\rangle$ represents the mean (macroscopic) position of the IS referred to the position of the planar substrate. The $\mathrm{CW}$ spectrum is sampled through the mean square amplitude $\left\langle\left|\hat{\xi}_{\mathbf{q}}\right|^{2}\right\rangle$ for each wave vector $\mathbf{q}$. We have checked that all the Fourier components with the same modulus $q=|\mathbf{q}|$ give similar mean square amplitudes, and all these values are accumulated to achieve better statistics in $\left\langle\left|\hat{\xi}_{q}\right|^{2}\right\rangle$. In the ISM the IS $\xi\left(\mathbf{R}, q_{\max }\right)$ interpolates through the surface pivots as the minimal area surface with a fixed upper wave-vector cutoff, $q_{\max }$, which determines the allowed level of corrugation. Here we make the usual choice $q_{\max }=2 \pi / \sigma$, with the molecular size as the limit 
for the corrugations of the IS. The results with other interpolation schemes, based on Delaunay triangular tessellations and Voronoi terraces, are used in Sec. V to compare with the ISM.

In our previous work ${ }^{21}$ we have explored the same system as here, with both canonical and restricted grand-canonical simulations. We compared the descriptions in terms of the ISM mean film thickness, $\xi_{\text {IS }}$, and in terms of the usual Gibbs dividing surface definition with a film thickness, $\xi_{N}$, that reflects the total number of particles enclosed in the simulation box. We found that the ISM allows a high accuracy in the determination of the IS and therefore in the thickness of the adsorbed film. The fluctuations of the mean film thickness $\xi_{\text {IS }}$ are well described by a simple mesoscopic Hamiltonian, in the same spirit as we do here for the $\mathrm{CW}$ fluctuations of the wetting films. An important result of that previous work was the effective interfacial potential $\Phi(\xi)$, defined as the free energy per unit area of a liquid film with uniform thickness $\xi$ at coexistence with its vapor,

$$
\Phi(\xi)=f\left(\xi \mid \mu_{\mathrm{lv}}\right)=-\log \left[\mathcal{P}\left(\xi \mid \mu_{\mathrm{lv}}\right) /\left(\beta A_{0}\right)\right]+\mathrm{C},
$$

where $\mathcal{P}\left(\xi \mid \mu_{\mathrm{lv}}\right)$ is the probability distribution for the mean film thickness, obtained in the grand-canonical ensemble at the bulk coexistence chemical potential $\mu_{\mathrm{lv}}$. The constant $\mathrm{C}$, tied to the normalization of $\mathcal{P}(\xi \mid \mu)$, and the value of $\mu_{\mathrm{lv}}$ are obtained from the requirement that $\Phi(\xi)=0$ for large $\xi$.

The results for $\Phi(\xi)$ were obtained with both $\xi \equiv \xi_{\text {IS }}$ and $\xi \equiv \xi_{\mathrm{N}}$ (see Fig. 6 in Ref. 21). The main advantage of the ISM definition is that, for any film thickness above one monolayer $\left(\xi_{\text {IS }} \gtrsim \sigma\right)$, the effective potential presents a perfect exponential behavior,

$$
\Phi\left(\xi_{\mathrm{IS}}\right)=\Phi_{0} e^{-\lambda \xi_{\mathrm{IS}}},
$$

with $\beta \Phi_{0} \sigma^{2}=1.97$ (i.e., $\beta \Phi_{0} A_{0}=215.4$ ), and the exponential decay $\lambda \sigma=1.55$. The exponential constant is also obtained from the decay of the liquid-vapor density profile $\rho(z)$ toward the liquid bulk, so that $\lambda$ is clearly identified as the true inverse correlation length.

From the exponential decay $\lambda$ and the simulation value of the macroscopic surface tension $\beta \gamma_{0} \sigma^{2}=0.66 \pm 0.01$, we get the so-called wetting parameter, which controls the critical behavior of the wetting layer: ${ }^{1,5}$

$$
\omega=\frac{k_{\mathrm{b}} T \lambda^{2}}{4 \pi \gamma_{0}}=0.29,
$$

a value similar to the effective one extracted from simulations for the Ising model. ${ }^{24}$ With this value of $\omega$ we expect only slight deviations of the MF predictions.

\section{THEORY}

The theoretical framework to study the fluctuations of liquid films adsorbed on walls is the mesoscopic interfacial effective Hamiltonian $\mathcal{H}[\xi]$, as a functional of the instantaneous shape of the interface $z=\xi(\mathbf{R}) \equiv \xi(x, y)$, which is the relevant collective parameter in the study of the wetting transitions. Within the Fourier description of the IS, (2), the interfacial Hamiltonian becomes a function of the Fourier components $\hat{\xi}_{q}$. For nonstructured substrates (such as the one studied here) the components with $q \neq 0$ have null mean value, $\left\langle\hat{\xi}_{q}\right\rangle=0$, so that the only relevant parameter in a MF description is the $q=0$ component, which gives the mean thickness of the wetting layer, $z=\langle\xi(\mathbf{R})\rangle \equiv \xi_{\text {IS }}$. The MF Hamiltonian is directly given by the effective interfacial potential, (4):

$$
\mathcal{H}_{\mathrm{MF}}[\xi]=A_{0} \Phi\left(\xi_{\mathrm{IS}}\right) \text {. }
$$

Beyond the MF description, the dependence of $\mathcal{H}[\xi]$ on $\hat{\xi}_{q}$ describes the $\mathrm{CW}$ fluctuations of the interface, and it is the subject of the theoretical analysis based on the RG theory. ${ }^{25,26}$ The simplest theoretical hypothesis splits the surface Hamiltonian into two independent contributions, $\mathcal{H}[\xi(\mathbf{R})]=\mathcal{H}_{\text {wall }}[\xi(\mathbf{R})]+\mathcal{H}_{\mathrm{CW}}[\xi(\mathbf{R})]$. The first one describes the interaction of the wetting film with the inert substrate in terms of the local effective potential,

$$
\frac{\mathcal{H}_{\text {wall }}[\xi(\mathbf{R})]}{A_{0}}=\int \frac{d^{2} \mathbf{R}}{A_{0}} \Phi(\xi(\mathbf{R})),
$$

averaged over the substrate area. The second contribution considers the corrugations of the film in terms of the classical CW Hamiltonian for a free liquid-vapor surface,

$$
\begin{aligned}
\frac{\mathcal{H}_{\mathrm{CW}}^{\text {class }}[\xi(\mathbf{R})]}{A_{0}} & =\gamma_{0} \int \frac{d^{2} \mathbf{R}}{A_{0}}\left[\sqrt{1+|\nabla \xi(\mathbf{R})|^{2}}-1\right] \\
& =\int \frac{d^{2} \mathbf{R}}{A_{0}}\left(\frac{\gamma_{0}}{2}|\nabla \xi(\mathbf{R})|^{2}+\mathcal{O}^{4}[\nabla \xi]\right),
\end{aligned}
$$

where the (macroscopic) surface tension $\gamma_{0}$ multiplies the (instantaneous) increase in area produced by the CW fluctuations on the edge of the film. The leading effects of the CW fluctuations are included with the expansion of (7) and (8) in powers of $\left|\hat{\xi}_{q}\right|^{2}$,

$$
\frac{\mathcal{H}\left[\hat{\xi}_{q}\right]}{A_{0}} \approx \Phi\left(\hat{\xi_{0}}\right)+\frac{1}{2} \sum_{q}\left(\Phi^{\prime \prime}\left(\hat{\xi_{0}}\right)+\gamma_{0} q^{2}\right)\left|\hat{\xi}_{q}\right|^{2}+\mathcal{O}\left(\left|\hat{\xi}_{q}\right|^{4}\right),
$$

so that, neglecting the quartic-order terms, each $\mathrm{CW}$ mode becomes an independent harmonic oscillator. The equipartition theorem gives

$$
\frac{A_{0}}{2}\left(\Phi^{\prime \prime}\left(\xi_{\text {IS }}\right)+\gamma_{0} q^{2}\right)\left\langle\left|\xi_{q}\right|^{2}\right\rangle=\frac{k_{b} T}{2}
$$

and the mean square amplitude is a Lorentzian function of the wave vector:

$$
\left\langle\left|\hat{\xi}_{q}\right|^{2}\right\rangle=\frac{k_{b} T}{\left(\Phi^{\prime \prime}\left(\xi_{\text {IS }}\right)+\gamma_{0} q^{2}\right) A_{0}} .
$$

The CW divergence at low $q$, predicted for free liquid surfaces, becomes limited by the effect of the substrate through the second derivative of the interfacial potential. Note that (11) is a direct generalization of the gravity effect on the $\mathrm{CW}$ amplitude, with $\Phi_{\text {grav }}(\xi)=\rho m g \xi^{2} / 2$, that sets the capillary length $l_{\text {grav }}=$ $\left[\gamma_{0} / \rho m g\right]^{1 / 2}$, in the range of millimeters, as the limit for the transverse size effect in the surface fluctuations for simple fluids. ${ }^{9}$ Similarly, the effective wall potential $\Phi\left(\xi_{\text {IS }}\right)$ in Eq. (11) sets a transverse correlation length $l_{\text {wall }}\left(\xi_{\text {IS }}\right)=\left[\gamma_{0} / \Phi^{\prime \prime}\left(\xi_{\text {IS }}\right)\right]^{1 / 2}$ that increases exponentially with film thickness. ${ }^{27-29}$ In our system, when the wetting film has two molecular layers, $\xi_{\text {IS }}=2 \sigma$, we have $l_{\text {wall }}\left(\xi_{\text {IS }}\right) \approx 3 \sigma$, while for four adsorbed layers we have $l_{\text {wall }}\left(\xi_{\text {IS }}\right) \gtrsim 13 \sigma$, and the $\mathrm{CW}$ fluctuations $\left\langle\xi(\mathbf{R})^{2}\right\rangle-\langle\xi(\mathbf{R})\rangle^{2}$ observed in our simulations are already 
restricted by the finite simulation transverse area $A_{0}=L_{x}^{2}<$ $l_{\text {wall }}^{2}$. To include all the relevant CW modes in a thick film of 10 layers we would need a simulation box with a transverse size of several thousand molecular diameters.

The role of the theories based on the surface Hamiltonian $\mathcal{H}[\xi(x, y)]$ is to fill the gap between the nanometer scale, explored with molecular simulations or density functional theories, ${ }^{30}$ and the behavior of thick films for transverse sizes in the range of $L_{x} \sim 10-100 \mu$, when all the relevant $\mathrm{CW}$ modes are included. The RG analysis ${ }^{25,26}$ shows that small effects beyond the simplest hypothesis (7)-(9) become relevant in the RG flux, so that they have to be included in the starting $\mathcal{H}[\xi(\mathbf{R})]$. The aim of this work is to extract the functional form of $\mathcal{H}[\xi]$ directly from the MC simulation results for $\left\langle\left|\hat{\xi}_{q}\right|^{2}\right\rangle$, as a function of the mean thickness $\xi_{\text {IS }}$ and the wave vector $q$. To that end, it is crucial to get a firm hold on the nanometric range, i.e., $q \sigma \sim 1$, and for wetting films that are not too thick, $\xi_{\text {IS }} \lesssim 4 \sigma$, so that the deviations of $\mathcal{H}[\xi]$ from the simplest functional form, (9), may appear clearly, over the unavoidable noise of the computer simulation. Therefore, although our simulations cover a wide range of wetting layer thicknesses $\sigma \lesssim \xi_{\text {IS }} \lesssim 10 \sigma$, the most important results come from the fluctuations of relatively thin films, which may be analyzed in terms of the theoretical Hamiltonians predicted from the limit of thick films.

It is well established ${ }^{17,20,31}$ that, when applied to the free liquid-vapor interface [i.e., $\Phi(\xi)=0$ ], prediction (11) is correct in the low $-q$ limit, for $q \sigma \ll 1$. However, in the nanoscale regime, $q \sigma \gtrsim 0.5$, accessible to a typical simulation, the observed mean square $\mathrm{CW}$ amplitude $\left\langle\left|\xi_{q}\right|^{2}\right\rangle$ starts to deviate from the simplest CWT prediction,

$$
\left\langle\left|\xi_{q}\right|^{2}\right\rangle_{\mathrm{LV}}=\frac{k_{b} T}{\gamma_{0} q^{2} A_{0}} .
$$

The deviation is usually described through the definition of a wave-vector-dependent surface tension, ${ }^{31,32}$ defined as

$$
\gamma_{\mathrm{LV}}(q) \equiv \frac{k_{b} T}{q^{2} A_{0}\left\langle\left|\xi_{q}\right|^{2}\right\rangle_{\mathrm{LV}}}
$$

and with a low- $q$ expansion,

$$
\gamma_{\mathrm{LV}}(q) \approx \gamma_{0}+\kappa q^{2}+\cdots
$$

We have to point out that long-range van der Waals interactions (not considered here) would produce a nonanalytical dependence $\gamma_{\mathrm{LV}}(q) \approx \gamma_{0}+a q^{2} \log (q / b)+\cdots$, so that the effective bending constant becomes divergently negative for low $q .{ }^{33}$ Those long-range interactions would also produce a power-law decay of $\Phi(\xi)$ and change the whole RG analysis of the problem.

In geometrical terms, the coefficient $\kappa$ in Eq. (14) represents a bending modulus that gives the free energy cost of curvature in a fixed area. In the generalized version of the CWT the integrand in Eq. (8), which is a local function of the gradient $\nabla \xi(\mathbf{R})$, is extended to be a function $\Phi_{\mathrm{CW}}^{\mathrm{gen}}\left(\nabla \xi, \nabla^{2} \xi, \ldots\right)$, that depends on higher order derivatives of $\xi(\mathbf{R})$, to include the effect of curvature. The expansion up to quadratic order in the $\mathrm{CW}$ amplitude, and the usual symmetry arguments for a (macroscopically) planar interface, lead to

$$
\begin{aligned}
\frac{\mathcal{H}_{\mathrm{CW}}^{\mathrm{gen}}[\xi]}{A_{0}} & =\int \frac{d^{2} \mathbf{R}}{A_{0}} \Phi_{\mathrm{CW}}^{\mathrm{gen}}\left(\nabla \xi(\mathbf{R}), \nabla^{2} \xi(\mathbf{R}), \ldots\right) \\
& \approx \int \frac{d^{2} \mathbf{R}}{A_{0}}\left(\frac{\gamma_{0}}{2}|\nabla \xi(\mathbf{R})|^{2}+\frac{\kappa}{2}\left|\nabla^{2} \xi(\mathbf{R})\right|^{2}+\cdots\right) \\
& =\sum_{q>0}\left(\gamma_{0}+\kappa q^{2}+\cdots\right) q^{2} \frac{\left|\hat{\xi}_{q}\right|^{2}}{2} \\
& \equiv \sum_{q>0} \gamma_{\mathrm{LV}}(q) q^{2} \frac{\left|\hat{\xi}_{q}\right|^{2}}{2}
\end{aligned}
$$

consistent with (13). Therefore, the function $\gamma_{\mathrm{LV}}(q)$ represents all the relevant aspects of the generic function $\Phi_{\mathrm{CW}}^{\mathrm{gen}}$, and its $q=0$ limit recovers the macroscopic limit $\gamma_{\mathrm{LV}}(0)=\gamma_{0}$ used in Eq. (8). The bending modulus $\kappa$, or the generic rise in $\gamma_{\mathrm{LV}}(q)$ as $q$ increases, may replace the need for an empirical cutoff for the $\mathrm{CW}$ wavelengths, $\lambda_{\mathrm{cw}}=2 \pi / q \geqslant \Lambda \equiv 2 \pi / q_{\mathrm{u}}$, imposed over the simplest Hamiltonian form, (9).

The functional form, (7), for the interaction of the wetting film with the substrate $\mathcal{H}_{\text {wall }}[\xi]$ may also be generalized, in the same way that $\mathcal{H}_{\mathrm{CW}}^{\text {gen }}[\xi]$ in Eq. (15) generalizes $\mathcal{H}_{\mathrm{CW}}^{\text {class }}[\xi]$ in Eq. (8). The function $\Phi_{\text {wall }}(\xi(\mathbf{R}))$ may be extended to $\Phi_{\text {wall }}^{\text {gen }}\left(\xi(\mathbf{R}), \nabla \xi(\mathbf{R}), \nabla^{2} \xi(\mathbf{R}), \ldots\right)$ to allow for the dependence on the local shape of the IS, within the range of the wall potential with the same expansion used in Eq. (15), we get

$$
\begin{aligned}
& \frac{\mathcal{H}_{\text {wall }}^{\text {gen }}[\xi]}{A_{0}} \\
& =\int \frac{d^{2} \mathbf{R}}{A_{0}} \Phi_{\text {wall }}^{\text {gen }}\left(\xi(\mathbf{R}), \nabla \xi(\mathbf{R}), \nabla^{2} \xi(\mathbf{R}), \ldots\right) \\
& \approx \int \frac{d^{2} \mathbf{R}}{A_{0}}\left(\Phi(\xi(\mathbf{R}))+\frac{\Delta \gamma(\xi(\mathbf{R}))}{2}|\nabla \xi(\mathbf{R})|^{2}\right. \\
& \left.\quad+\frac{\Delta \kappa(\xi(\mathbf{R}))}{2}\left|\nabla^{2} \xi(\mathbf{R})\right|^{2}+\cdots\right)=\Phi\left(\hat{\xi_{0}}\right) \\
& \quad+\sum_{q>0}\left(\Phi^{\prime \prime}\left(\hat{\xi_{0}}\right)+\Delta \gamma\left(\hat{\xi_{0}}\right) q^{2}+\Delta \kappa\left(\hat{\xi_{0}}\right) q^{4}+\cdots\right) \frac{\left|\hat{\xi}_{q}\right|^{2}}{2} \\
& \equiv \Phi\left(\hat{\xi_{0}}\right)+\sum_{q>0}\left(\Phi^{\prime \prime}\left(\hat{\xi_{0}}\right)+\Delta \gamma\left(q, \hat{\xi_{0}}\right) q^{2}\right) \frac{\left|\hat{\xi}_{q}\right|^{2}}{2} .
\end{aligned}
$$

The functional dependence of $\mathcal{H}_{\text {wall }}^{\text {gen }}[\xi]$, up to quadratic order in the amplitude of the $\mathrm{CW}$ fluctuations, is given by the functions $\Phi\left(\xi_{\text {IS }}\right)$ and $\Delta \gamma\left(q, \xi_{\text {IS }}\right)$. The first one, (4), has already been obtained, ${ }^{21}$ and the second may be directly extracted from the mean square $\mathrm{CW}$ amplitude, as a direct generalization of (11),

$$
\left\langle\left|\hat{\xi}_{q}\right|^{2}\right\rangle=\frac{k_{b} T}{\left(\Phi^{\prime \prime}\left(\xi_{\mathrm{IS}}\right)+\left(\gamma_{\mathrm{LV}}(q)+\Delta \gamma\left(q, \xi_{\mathrm{IS}}\right)\right) q^{2}\right) A_{0}} .
$$

We should remark that the bottom line in Eqs. (15) and (16), in terms of $\gamma_{\mathrm{LV}}(q)$ and $\Delta \gamma\left(q, \xi_{\text {IS }}\right)$, are more general than the top lines in the same equations, given in terms of the local function of $\xi(x, y)$ and its derivatives. Strictly nonlocal interfacial Hamiltonians, as proposed by Parry and 
coworkers, ${ }^{34}$ include double integrals over the surface, like

$$
\mathcal{H}_{\mathrm{nl}}[\xi] \sim \int d^{2} \mathbf{R}_{1} S\left(\xi\left(\mathbf{R}_{1}\right)\right) \int d^{2} \mathbf{R}_{2} S\left(\xi\left(\mathbf{R}_{2}\right)\right) K\left(R_{12}\right),
$$

where a kernel function $K\left(\left|\mathbf{R}_{1}-\mathbf{R}_{2}\right|\right)$ links the functional dependence at two points of the interface, with a generic function $S(\xi)$. The expansion of $\mathcal{H}_{\mathrm{nl}}[\xi]$ in terms of $\xi\left(\mathbf{R}_{2}\right) \approx$ $\xi\left(\mathbf{R}_{1}\right)+\nabla \xi\left(\mathbf{R}_{1}\right)\left(\mathbf{R}_{2}-\mathbf{R}_{1}\right)+\cdots$ may not converge (if there are important changes of the film thickness over the range of the kernel) and, in that case, $\mathcal{H}_{\mathrm{nl}}[\xi]$ cannot be approximated by the first lines in Eq. (16). However, the expansion of $\mathcal{H}_{\text {nl }}[\xi]$ up to quadratic order in $\hat{\xi}_{q}$ would still have the form of the last line in Eq. (16). In that case, the function $\Delta \gamma\left(q, \xi_{\text {IS }}\right)$ would not converge as a polynomial expansion $\Delta \gamma\left(\xi_{\text {IS }}\right)+\Delta \kappa\left(\xi_{\text {IS }}\right) q^{2}+\cdots$.

Our direct evaluation of $\gamma_{\mathrm{LV}}(q)+\Delta \gamma\left(\xi_{\mathrm{IS}}, q\right)$ from the simulation results for $\left\langle\left|\hat{\xi}_{q}\right|^{2}\right\rangle$ does not presuppose any particular dependence with $q$, so that we are not assuming any particular form, local or nonlocal, for the interfacial Hamiltonian. On the other hand, the (generalized) wall contribution to the surface tension $\Delta \gamma\left(\xi_{\text {IS }}, q\right)$ has to vanish in the limit of very thick layers, and the most obvious trial form to fit the MC results would be the simple exponential function that gives $\Phi\left(\xi_{\text {IS }}\right)$ in Eq. (4), as extracted from grand-canonical MC simulations of the same model used here, ${ }^{21}$ i.e.,

$$
\Delta \gamma\left(q, \xi_{\mathrm{IS}}\right) \approx \Delta \gamma_{\mathrm{w}}(q) e^{-\lambda \xi_{\mathrm{IS}}}
$$

The Hamiltonian form used in the RG analysis by Fisher and $\operatorname{Jin}^{3}$ corresponds to (16) and (19) with a $q$-independent function $\Delta \gamma_{\mathrm{w}}(q) \approx \Delta \gamma_{\mathrm{FJ}}$. The simplest classical version, (7), is recovered if $\Delta \gamma_{\mathrm{FJ}}=0$. For flat substrates the Hamiltonian of Fisher and Jin is recovered as the low- $q$ limit of the nonlocal functional form proposed by Parry et al., ${ }^{34}$ but the latter includes a $q$ dependence in $\Delta \gamma\left(q, \xi_{\text {IS }}\right)$. Therefore, the first difference between the two theoretical assumptions would appear as the curvature terms in the expansion $\gamma_{\mathrm{LV}}(q)+\Delta \gamma\left(q, \xi_{\mathrm{IS}}\right) \approx$ $\gamma_{0}+\Delta \gamma_{\mathrm{FJ}} e^{-\lambda \xi_{\mathrm{IS}}}+\left(\kappa+\Delta \kappa\left(\xi_{\mathrm{IS}}\right)\right) q^{2}+\cdots$. Parry and Rascón 6 have recently shown that the nonlocal Hamiltonian also implies the wave-vector dependence of $\gamma_{\mathrm{LV}}(q)$. ISM results extract the value of $\kappa$ from computer simulation of the liquid surface, ${ }^{16}$ and the results presented in the next section aim to test (19) and to extract the possible curvature terms in the wall damping term $\Delta \gamma\left(q, \xi_{\text {IS }}\right)$ for wetting films.

\section{RESULTS}

Figures 1 and 2 present the MC results for the inverse mean square values $\Gamma\left(q, \xi_{\text {IS }}\right) \equiv\left\langle\left|\hat{\xi}_{q}\right|^{2}\right\rangle^{-1}$, as functions of the film thickness $\xi_{\text {IS }}$, for the seven lowest values of $q$ within our simulation box (from $q \sigma=0.6$ to 1.9 ). As expected, the amplitude of the $\mathrm{CW}$ fluctuations decreases ( $\Gamma$ increases) for larger $q$ (shorter wavelength) and for thinner films (damping by the wall). Within the accuracy of our results, $\Gamma\left(q, \xi_{\text {IS }}\right)$ is flat for $\xi_{\text {IS }} \gtrsim 5 \sigma$, and it recovers the prediction for the free liquid slab (filled circles), i.e., $\Gamma_{\mathrm{LV}}(q)=\gamma_{\mathrm{LV}}(q) q^{2} A_{0} /\left(k_{b} T\right)$, with $\gamma_{\mathrm{LV}}(q)$ defined in Eq. (13) and presented in Fig. 3(a). As reported previously, ${ }^{17,20}$ the extrapolation of the ISM results to the $q=0$ limit is consistent with the thermodynamic surface tension obtained in the same computer simulations, $\beta \sigma^{2} \gamma_{\mathrm{LV}}(0) \equiv \beta \sigma^{2} \gamma_{0}$, but the effect of the bending modulus is

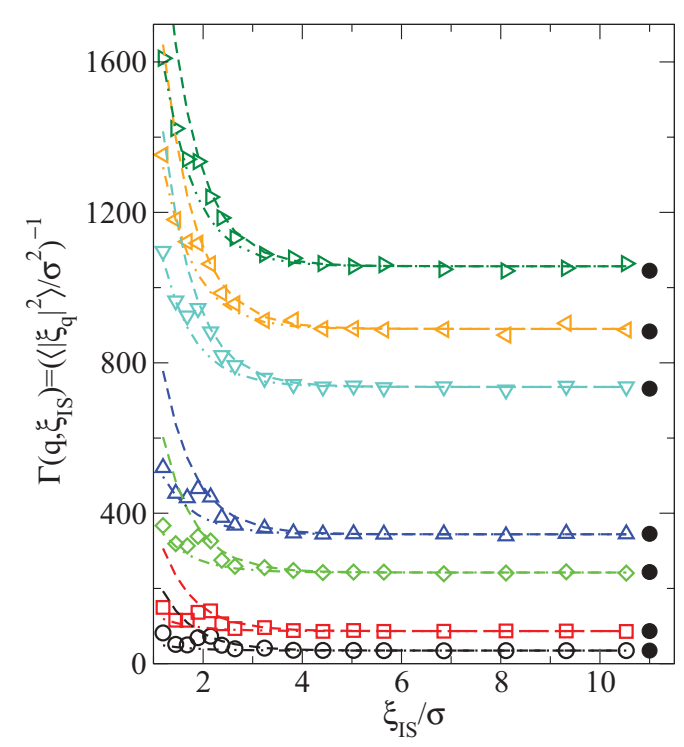

FIG. 1. (Color online) Inverse of the mean square amplitude of the intrinsic surface Fourier components $\Gamma\left(q, \xi_{\text {IS }}\right)$ versus the thickness of the adsorbed liquid film, for different values of $q$. Circles, $q \sigma=0.601$; squares, $q \sigma=0.850$; diamonds, $q \sigma=1.202$; downward triangles, $q \sigma=1.344$; upward triangles, $q \sigma=1.700 ;$ left triangles, $q \sigma=1.802$; and right triangles, $q \sigma=1.900$. Filled (black) circles represent the $\left(\left\langle\left|\xi_{q}\right|^{2}\right\rangle\right)^{-1}$ value of the free liquid slab. Dashed lines, fit to the upper envolvent; and dashed-dotted lines, fit to the lower envolvent. Both fits use the exponential decay, (21).

clearly observed in the increase in $\gamma_{\mathrm{LV}}(q)$ for $q \sigma \gtrsim 0.5$. At the largest wave vector, $q \sigma=1.90$, our results for $\Gamma\left(q, \xi_{\text {IS }}\right)$ are about four times larger than the predictions of the classical Hamiltonian, (7) and (8), mainly because of the large bending contribution to the surface tension of the free liquid surface, $\gamma_{\mathrm{LV}}(q) \approx 4 \gamma_{0}$. The classical prediction from (11) should be

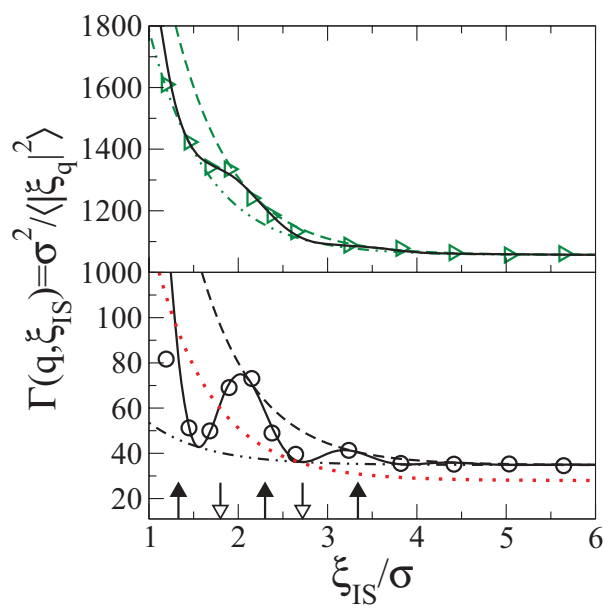

FIG. 2. (Color online) Detailed and enlarged view of the upper $(q \sigma=1.90$; top $)$ and lower $(q \sigma=0.601$; bottom $)$ curves in Fig. 1 . Dashed lines and symbols have the same meaning as in Fig. 1. The solid line is the fit to the decaying oscillatory function, (20). The dotted line shows the classical prediction, (11), at $q \sigma=0.601$. Arrows on the bottom axis give the positions of the minima (downward open arrows) and maxima (upward filled arrows) of the mean density profile for thick wetting layers. 
recovered in the low- $q$ limit, and the dotted line in the bottom panel in Fig. 2 shows that for $q \sigma=0.60$ it is already in the observed range of values. The difference in the plateau for $\xi_{\text {IS }} \gtrsim 5 \sigma$ represents the effect of the bending contribution at the free liquid surface, which is much smaller than for the larger $q$. However, the main difference between the classical prediction and our simulation results is the strong oscillatory structure of $\Gamma\left(q, \xi_{\text {IS }}\right)$ in films with $\xi_{\text {IS }} \lesssim 4 \sigma$, which is very well fitted by

$$
\begin{aligned}
& \frac{\Gamma\left(q, \xi_{\mathrm{IS}}\right)}{A_{0}} \\
& \quad \approx q^{2} \beta \gamma_{\mathrm{LV}}(q)+e^{-\lambda \xi_{\mathrm{IS}}}\left[B_{\text {mean }}+B_{\mathrm{osc}} \cos \left(q_{\mathrm{osc}}\left(\xi_{\mathrm{IS}}-z_{0}\right)\right)\right],
\end{aligned}
$$

assuming that the boundaries have the same exponentially decay $\lambda \sigma=1.55$ as the MF potential $\Phi\left(\xi_{\text {IS }}\right)$ in Eq. (4). The upper and lower bounds for $\Gamma\left(q, \xi_{\text {IS }}\right)$, represented in Figs. 1 and 2 , are given by

$$
B^{\text {(low })}(q) e^{-\lambda \xi_{\mathrm{IS}}} \leqslant \frac{\Gamma\left(q, \xi_{\mathrm{IS}}\right)}{A_{0}}-q^{2} \beta \gamma_{\mathrm{LV}}(q) \leqslant B^{(\mathrm{up})}(q) e^{-\lambda \xi_{\mathrm{IS}}}
$$

with the functions $B^{\text {(up, low) }}(q)=B_{\text {mean }} \pm B_{\text {osc }}$ shown in Figs. 3(b) and 3(c).

The theoretical prediction, (17), from the generalized surface Hamiltonian leads to

$$
\begin{aligned}
B^{(\text {low })}(q) & \leqslant \beta\left(\Phi^{\prime \prime}\left(\xi_{\text {IS }}\right)+q^{2} \Delta \gamma\left(q, \xi_{\text {IS }}\right)\right) e^{\lambda \xi_{\text {IS }}} \\
& =\beta\left(\lambda^{2} \Phi(0)+q^{2} \Delta \gamma\left(q, \xi_{\text {IS }}\right) e^{\lambda \xi_{\text {IS }}}\right) \leqslant B^{\text {(up })}(q) .
\end{aligned}
$$

The upper boundary $B^{\text {(up) }}(q)$ in Fig. 3(b) describes the wall effect for films that show the largest damping. The extrapolation to low $q$ is perfectly consistent with (22), i.e., $B^{\text {(up) }}(0) \approx \lambda^{2} \beta \Phi(0)=4.73 / \sigma^{4}$. Above that constant value, there is a nearly quadratic increase with $q$, consistent with the assumption of Fisher and Jin, ${ }^{3}$ i.e., $B^{\text {(up) }}(q) \approx \lambda^{2} \beta \Phi(0)+$ $\beta \Delta \gamma_{\mathrm{FJ}}^{\text {(up) }} q^{2}$, with only one free parameter to describe the wall damping on the CW fluctuations in Eq. (19) for any value of $q$ and for any film thickness. Its value $\beta \sigma^{2} \Delta \gamma_{w}^{\text {(up) }}(0) \approx 10.22$, extracted from our ISM, provides a firm quantitative link between the molecular simulations and the surface Hamiltonian used in the RG analysis. We may even characterize the small deviations of $B^{\text {up) }}(q)$ from a pure parabolic shape, allowing for a function $\Delta \gamma_{w}^{\text {(up) }}(q)=\Delta \gamma_{w}^{\text {(up) }}(0)+\Delta \kappa_{w}^{\text {(up) }} q^{2}+\cdots$. However, as is clearly seen in a comparison between Figs. 3(a) and 3(b), the relative importance of the wall bending term, $\Delta \kappa_{w}^{\text {(up) }} / \Delta \gamma_{w}^{\text {(up) }} \approx 0.06 / \sigma^{2}$, is much less than in the free surface liquid-vapor interface, $\kappa / \gamma_{0} \approx 0.80 / \sigma^{2}$. The bending (or nonlocal) effects in the fluctuating film are essentially similar to those in the free liquid-vapor interface.

Our ISM results for $\left\langle\left|\hat{\xi}_{q}\right|^{2}\right\rangle$, together with the previous simulation results for the MF wall potential $\Phi\left(\xi_{\text {IS }}\right)$ and the CW spectrum at the liquid surface $\gamma_{\mathrm{LV}}(q)$, appear to fit perfectly into the theoretical assumptions in Eq. (16). This is highly rewarding, however, we have to recall that $B^{\text {(up) }}(q)$ gives only the upper limit for $\Gamma\left(q, \xi_{\text {IS }}\right)$, i.e., the lower bound for the CW square amplitude $\left\langle\left|\hat{\xi}_{q}\right|^{2}\right\rangle$. The results for $B^{(\text {low })}(q)$, also presented in Fig. 3(c), break the apparent simplicity of the analysis. The function $B^{(\text {low })}(q)$ diverts from the parabolic behavior event at very low values of $q$ due to the high relative

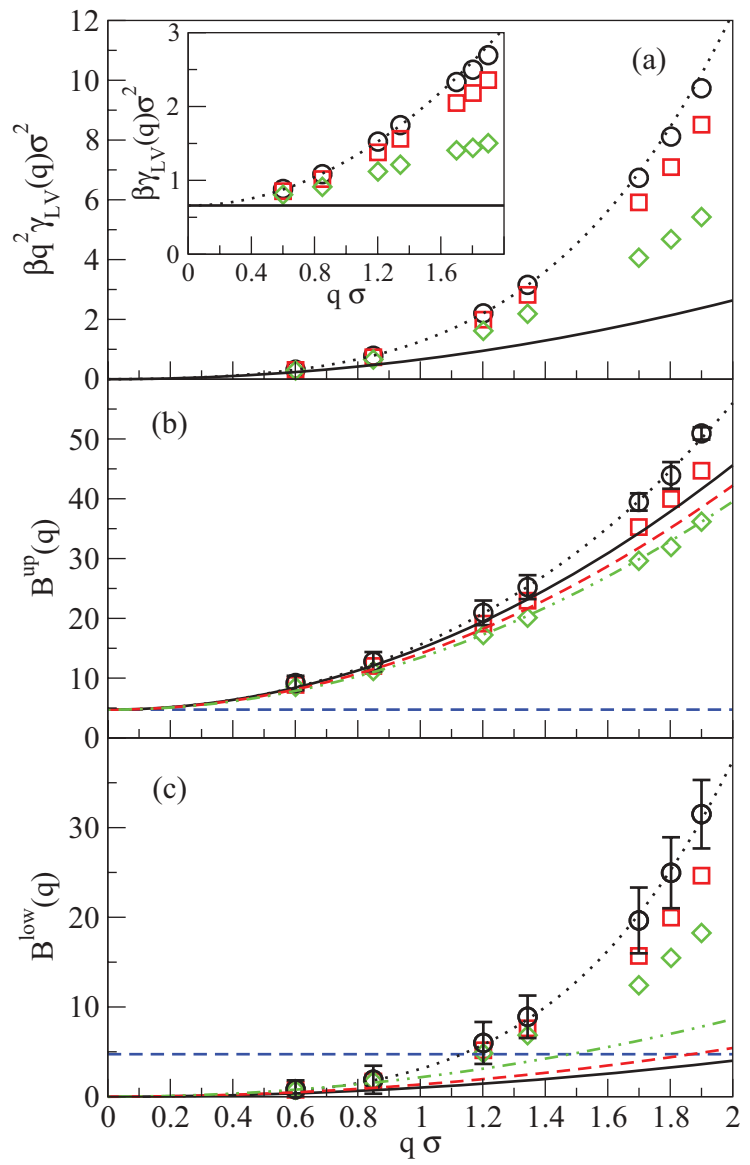

FIG. 3. (Color online) (a) The free liquid surface contribution $\left[q^{2} \beta \gamma_{\mathrm{LV}}(q)\right]$ to $\Gamma\left(q, \xi_{\text {IS }}\right)$. Inset: The effective $q$-dependent surface tension $\gamma_{\mathrm{LV}}(q)$ of the free liquid-vapor interface. (b), (c) Values of $B^{\text {up }}(q)$ and $B^{\text {low }}(q)$, respectively, as a function of $q$, obtained from the fit of $\Gamma\left(q, \xi_{\text {IS }}\right)$ in Fig. 1 to the decaying oscillatory function given by (20). Open (black) circles are the results obtained using the ISM intrinsic surface; open (red) squares, the Delaunay triangulated; and open (green) triangles, the terraced Voronoi. The quadratic fits at low $q$ are as follows: solid (black) lines, ISM surface; dashed (red) lines, Delaunay triangled; and dashed-dotted (green) lines, terraced Voronoi. Dotted lines are the fits of the ISM results to a quartic function: $\lambda^{2} \beta \Phi(0)+\beta \Delta \gamma q^{2}+\beta \Delta \kappa q^{4}$ in (b) and (c) and $\beta \gamma_{0} q^{2}+\beta \kappa q^{4}$ in (a). In (b) and (c) the horizontal dashed (blue) line is the constant value $B=\lambda^{2} \beta \Phi(0)$ predicted by the simple local Hamiltonian given by (9).

relevance of the bending term $\Delta \kappa_{w}^{\text {(low) }} / \Delta \gamma_{w}^{(\text {low })} \approx 2.0 / \sigma^{2}$. More important is that the low $q$ extrapolates to $B^{(\text {low })}(0) \approx 0$, rather than to the value $\lambda^{2} \Phi(0)=\Phi^{\prime \prime}(0)$ predicted by (22). Note that, without the wall contribution $\Phi^{\prime \prime}(0)$, the CW fluctuations in the film would diverge in the $q \rightarrow 0$ limit, as in the free liquid surface.

The oscillatory behavior of $\Gamma\left(q, \xi_{\text {IS }}\right)$, and hence of the difference between $B^{\text {(up) }}(q)$ and $B^{(\text {low })}(q)$, comes clearly from the formation of the molecular layers in the density profile $\rho(z)$ of the adsorbed film. The wave-vector parameter in the fit, $(20)$, is $q_{\mathrm{osc}} \approx 2 \pi / \sigma$, i.e., in the range for molecular layering oscillations. The arrows on the bottom axis in Fig. 2 mark the positions of the IS $\xi_{\text {IS }}=z_{\max \text { min }}$, where the density profile $\rho(z)$ develops maxima and minima; and there is a 


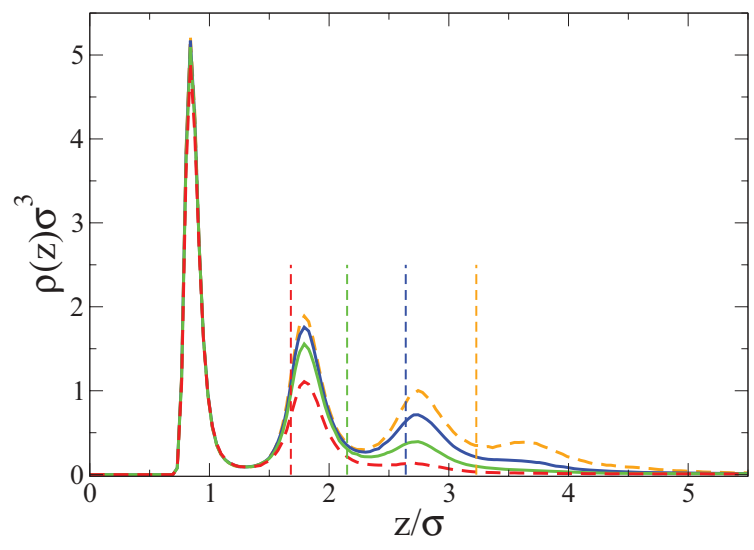

FIG. 4. (Color online) Mean density profiles for wetting films in simulation with $N=200-350$ particles $N$. Dark dashed (red) line, $N=200$; solid gray (green) line, $N=250$; dark solid (blue) line, $N=300$; and light dashed (orange) line:, $N=350$. Vertical dashed lines point out the mean positions of the intrinsic surfaces $\xi_{\text {IS }}=$ $1.68 \sigma, 2.15 \sigma, 2.64 \sigma$, and $3.23 \sigma$, respectively.

clear correlation with the oscillations of $\Gamma\left(q, \xi_{\text {IS }}\right)$. In Fig. 4 we present the density profiles $\rho(z)$ for films with $1.5 \sigma \lesssim$ $\xi_{\text {IS }} \lesssim 3.5 \sigma$, covering two full oscillations of $\Gamma\left(q, \xi_{\text {IS }}\right)$. These profiles are far from the theoretical image, which supposes that the external edge of the film is similar to a liquid-vapor surface. However, these wetting films are within the range of interest to analyze the possible nonlocal effects in the interfacial Hamiltonian. Thicker films $\left(\xi_{\text {IS }} \gtrsim 8 \sigma\right)$ present density profiles with a liquid-like plateau between the wall and the outer edge, but for them the wall damping effect on the $\mathrm{CW}$ fluctuations is too weak, compared with the intrinsic noise of the simulation results. With very large simulation boxes (to access at very low $q$ ), we might enhance the wall effects for thick films, but that would take us to the range $q \sigma \ll 1$, where the simplest local Hamiltonian, (7) and (8), is very accurate, and we could not discern any nonlocal effect beyond it. Moreover, in our previous work ${ }^{21}$ we have observed that, even for very thin films, the surface potential $\Phi\left(\xi_{\text {IS }}\right)$ extracted by the ISM was accurately described by the smooth exponential, (4), with the predicted decay length and without any apparent effect of the strong density layering near the wall. The excellent agreement of our results for $B^{\text {(up) }}(q)$ and the established theoretical description from (16) also suggests that the fluctuations of these strongly layered films may also be very well described in terms of the same exponential decay. This is probably the most important result of this work.

We discussed in our previous work ${ }^{21}$ that the apparent irrelevance of the strong layering in the smooth function $\Phi\left(\xi_{\text {IS }}\right)$ implies that the structure in $\rho(z)$ is a projection of the inherent correlation structure in the liquid, rather than a strong perturbation of that structure. The ISM extracts intrinsic density profiles at the liquid-vapor interface that are as structured as the usual (mean) density profiles near a planar wall; observing the layering at the inner or at the outer edge of the film is just a matter of choice for the representation of the molecular distances, either taking the $z_{0}=0$ position of the wall or the $z_{0}=\xi(\mathbf{R})$ position of the IS as the origin to represent the positions $z_{i}-z_{0}$ of each particle $(i=1, \ldots, N)$.
The enhanced fluctuations described by $B^{(\text {low })}(q)$ represent the frustration, or negative interference, between the mean density layering with respect to the wall and the intrinsic layering with respect to the outer edge of the wetting film, which appears to be a tendency of the system to undergo some layering transitions. Indeed, if the growth of the molecular layers were discrete, the function $\Phi\left(\xi_{\text {IS }}\right)$ should show a piece-like structure, with the coexisting values of the mean thickness joined by straight lines, i.e., $\Phi^{\prime \prime}\left(\xi_{\text {IS }}\right)=0$, as apparently deduced from $B^{(\text {low })}(q)$ in Fig. 3(c). The observation of such behavior in the canonical MC simulations analyzed here, but not in the (restricted) grand-canonical MC used to get $\Phi\left(\xi_{\text {IS }}\right),{ }^{21}$ suggests that the oscillatory structure of $\Gamma\left(q, \xi_{\text {IS }}\right)$ could be a finite-size effect, producing a spurious layering transition. With this interpretation the results for $B^{\text {(up) }}(q)$, i.e., the bottom line for the $\mathrm{CW}$ mean square amplitude, could represent the true Hamiltonian $\mathcal{H}_{\text {wall }}[\xi(\mathbf{R})]$.

\section{DISCUSSION OF THE DEFINITION OF THE INTRINSIC SURFACE}

We must consider a problem that has already appeared in the description of the $\mathrm{CW}$ spectrum for a free liquid surface. The only physical reality at the liquid interface is the discrete set of molecular positions. The mathematical surface $z=\xi(\mathbf{R})$ that defines the instantaneous IS is a useful construction since it keeps the relevant fluctuations in the long-CW limit. However, as we push the interpretation of $\xi(\mathbf{R})$ to corrugations at the mesoscopic range, there are unavoidable ambiguities, since there is not a unique way to define the boundary for the liquid phase. It has often been assumed that the geometrical aspects of the surface, relevant in the long-wavelength limit, should not be affected by this problem. Indeed, very different definitions of the smooth surface $z=\xi(\mathbf{R})$, to be associated with the same molecular configuration, may give the same mean square gradient $\left\langle|\nabla \xi(\mathbf{R})|^{2}\right\rangle$ ), i.e., the same mean value for the IS area in Eq. (8), when averaged over the equilibrium configurations along a computer simulation. However, the specific details of the interpolation scheme become relevant to estimate the mean square curvature (or $\left\langle\left|\nabla^{2} \xi(\mathbf{R})\right|^{2}\right\rangle$ ). Therefore, the bending rigidity $\kappa$, which weights the curvature contribution to the surface Hamiltonian $\mathcal{H}[\xi]$, is also dependent on the specific definition for the IS. ${ }^{32,35}$ Any experimental measurement, theoretical prediction, or computer simulation evaluation of $\kappa$ is as good as the definition of $\xi(\mathbf{R})$ that is behind it.

Over the last decade, the exploration of $\xi(\mathbf{R})$ in computer simulations has built a corpus of practical definitions that give $\kappa$ within a narrow range of values, particularly when the details in the definition of the IS are optimized to give the best simultaneous representation of different physical aspects, like the layering structure, the interfacial kinetics, and the hydrodynamics of the CW modes. ${ }^{16}$ Moreover, when we use different definitions (within that corpus) we observe that the variation of physical parameters, like the temperature or the range of the potential, always produce very similar changes in $\gamma_{\mathrm{LV}}(q)$, particularly in $\kappa$. In this context, we have explored how the results for $\Delta \gamma\left(q, \xi_{\text {IS }}\right)$ presented in the previous section may be affected by the specific choice used in the definition of the surface $z=\xi(\mathbf{R})$ from the instantaneous molecular positions in the $\mathrm{MC}$ simulation. 
To be valid down to the molecular level, the definition of the IS definition has to be pinned to the molecules at the surface, identified with a percolation analysis, which is computationally costly and theoretically awkward. Any simpler definition, like a local Gibbs dividing surface based on a density balance across the interface, is doomed to failure at scales of $q \sigma \sim 1$. Even within the percolation analysis, we have to choose specific definitions and parameters, which are reflected in the optimal number of surface pivots per unit area, $n_{s}$, selected by the $\operatorname{ISM}^{16}$ and in the specific interpolation scheme to get $\xi(\mathbf{R})$ from that set of pivots.

In the original version of the ISM, the surface $z=\xi(\mathbf{R})$ was defined as the minimal area surface, going exactly through the positions of the selected surface pivots and having a strict upper limit $q \leqslant q_{u} \approx 2 \pi / \sigma$ in its Fourier representation, (2). An alternative representation, has been used more recently, ${ }^{16,36}$ with a Delaunay triangulation of the pivot coordinates on the $(x, y)$ plane, to identify the nearest neighbors of each pivot. Then the surface $z=\xi(\mathbf{R})$ is made of planar triangular facets, joining the edges of nearest-neighbor pivots. A third definition is the terraced Voronoi surface, ${ }^{37}$ in which the projection of the pivots onto the $(x, y)$ plane is followed by a Voronoi tessellation, i.e., for each projected pivot we define its Voronoi cell as all points whose distance to the given projected pivot is not greater than the distance to the other projected pivots. The terraced Voronoi surface is defined assigning to all the points belonging to one Voronoi cell the height of its associated pivot. For the last two procedures the Fourier components of the $\mathrm{CW}$ spectrum are obtained by fitting the faceted or terraced surfaces to (2). Note that in all cases the CW amplitudes $\hat{\xi}_{q}$ are the Fourier components of the corresponding surface, and not those of the discrete set of pivots, which would lead to spurious $\gamma_{\mathrm{LV}}(q)$, because the bulk-like molecular correlations along the transverse directions would be mixed with the surface correlations induced by the CW. For the typical molecular configurations, the aspect of the ISM and the Delaunay triangulated surfaces is very similar. They only differ in the sharp edges between the facets, which appear at length scales well below the molecular diameter $\sigma$. In contrast, the terraced surface is rather different and apparently unrealistic at the scale of $\sigma$, with the abrupt changes between horizontal terraces. Comparison among the results of these three definitions for the IS allows us to check the soundness of the whole approach.

The results for $q^{2} \gamma_{\mathrm{LV}}(q), B^{\text {(up) }}(q)$, and $B^{\text {(low) }}(q)$ presented in Fig. 3 confirm our expectations. All these functions are independent of the definition of $\xi(\mathbf{R})$ up to the quadratic order $\sim q^{2}$, while the results at $q^{4}$ and higher orders have similar qualitative behaviors but different quantitative values. In general, the ISM gives the largest bending terms, closely followed by the Delaunay triangulation. The terraced Voronoi surfaces give null bending contribution in $B^{\text {(up) }}(q)$, while for the free liquid surface $\kappa$ is less than half the value obtained from the ISM. Similar changes in $\kappa$ appear within the ISM definition if we reduce the number of surface pivots per unit area $n_{s}$ to slightly below its optimal value. ${ }^{16}$ Therefore, the surface tension terms, $\gamma_{\mathrm{LV}}(0)=\gamma_{0}$ and $\Delta \gamma_{w}^{\text {(up,low) }}(0)$, are robust, while the bending coefficients, $\kappa$ and $\Delta \kappa_{w}^{\text {(up, low) }}$, depend on the specific definition of the IS. Within the restricted class of definitions used here, that dependence is mild, e.g., the relative value of the bending modulus with respect to the surface tension may be estimated to be in the range $0.4 / \sigma^{2} \lesssim \kappa / \gamma_{0} \lesssim 0.8 / \sigma^{2}$ for the free liquid surface and $0 \lesssim \Delta \kappa_{w} / \Delta \gamma_{w} \lesssim 0.07 / \sigma^{2}$ for the wall damping term. The qualitative behavior of the $\mathrm{CW}$ spectrum at the wetting film would not change within these ranges; there is a clear bending (or nonlocal) effect that is essentially given by the free liquid surface. The contribution of these effects to $\mathcal{H}_{\text {wall }}[\xi]$ is much smaller, and it falls within the inherent uncertainty of the mesoscopic description.

We should not forget that other apparently reasonable definitions of the IS (e.g., the local Gibbs dividing surface) lead to very different results for $\gamma_{\mathrm{LV}}(q)$, with negative bending terms. This is produced by a poor separation of the bulk-like density fluctuations near the surface, which are interpreted as enhanced CW fluctuations. ${ }^{10,38}$

Therefore, to distinguish among different theoretical proposals, local or nonlocal, for the surface Hamiltonian $\mathcal{H}[\xi]$, we have to bear in mind that any contribution beyond the square gradient term in Eqs. (15) and (16) would reflect the (explicit or implicit) definition of the IS.

\section{CONCLUDING REMARKS}

In this work we have studied the $\mathrm{CW}$ fluctuations at the edge of wetting layers and how they depend on the mean film thickness $\xi_{\text {IS }}$. We use MC computer simulations of a simple but realistic model of the wall-fluid interface and the method known as the ISM $^{16,17}$ to extract the shape of the IS $z=\xi(\mathbf{R})$ directly from the molecular positions. This is an important difference from any previous analysis of $\mathrm{CW}$ fluctuations in wetting layers, which were done relying on the observation of density-density surface correlations. ${ }^{8}$ The subtleties in the connection between the fluctuations of the IS and those of the fluid density near the surface have often been ignored as being irrelevant for the macroscopic limit. However, they are crucial if we want to explore the mesoscopic range in which the effective interfacial Hamiltonian $\mathcal{H}[\xi]$ may differ from the simplest CWT with a local exponential wall potential, (7) and (8). Our explicit sampling of $\xi(\mathbf{R})$ may be directly compared with the predictions of any approximation for $\mathcal{H}[\xi]$, without the (uncontrolled) approximations to go from $\left\langle\left|\hat{\xi}_{q}\right|^{2}\right\rangle$ to the density-density correlations. Moreover, we have checked the influence of the specific definition to get $\xi(\mathbf{R})$ from the molecular positions, which are the only physical reality described by $\mathcal{H}[\xi]$.

We have analyzed wetting layers with thicknesses between 1 and 10 monolayers, but only those with $\xi_{\text {IS }} \lesssim 4 \sigma$ give relevant information about the damping effect of the substrate wall on CW fluctuations. Using much larger simulation boxes we could access smaller wave vectors, which would show the influence of the wall in thicker films, but then the results would be trivially reproduced by the simplest local version of $\mathcal{H}[\xi]$. The possible effects of surface bending, (15) and (16), or nonlocal dependence, (18), have to be explored in the range of $q \sigma \sim 1$ for wave vectors and for relatively thin films. In realistic models these thin films have strongly layered density profiles $\rho(z)$, and their influence on the CW spectrum has to be tested. In a previous paper ${ }^{21}$ we reported that the wall potential $\Phi\left(\xi_{\text {IS }}\right)$ extracted by our method in (restricted) 
grand-canonical MC simulations was a smooth exponential function of the mean film thickness, despite the fact that $z=\langle\xi(\mathbf{R})\rangle$ had to cross over the strong layers in $\rho(z)$. In the present work we have used canonical MC simulations of the same system to measure the $\mathrm{CW}$ fluctuations with respect to the best possible determination of the mean film thickness, and we get a clear influence of the layering structure in the mean square amplitude of the CWs.

Along the line of films with the maximum damping effect of the wall (i.e., the upper boundary for the inverse of $\left\langle\left|\hat{\xi}_{q}\right|^{2}\right\rangle$ shown in Figs. 1-3), our results closely follow the predictions from the theoretical models for $\mathcal{H}[\xi]$. From layer to layer, the dependence on the film thickness is given by the same exponential decay, $\sim e^{-\lambda \xi_{\text {IS }}}$, as the potential $\Phi\left(\xi_{\text {IS }}\right)$, where the decay constant $\lambda$ is the inverse correlation length in the bulk liquid. The dependence on $q$ fits well into the form $\Phi^{\prime \prime}\left(\xi_{\text {IS }}\right)+q^{2} \Delta \gamma_{w}(0) e^{-\lambda \xi_{\text {IS }}}$ used in the RG analysis by Fisher and Jin, ${ }^{3}$ but the nonlocal effects pointed out by Parry et al. ${ }^{4,5}$ appear to be strong and clear in the contribution $\gamma_{\mathrm{LV}}(q)$, independent of $\xi_{\text {IS }}$, which gives the free liquid surface limit for thick films. In this respect, our method to extract the CW fluctuations from the molecular positions gives a quantitative and testable link between the molecular and the mesoscopic descriptions of wetting films. From the molecular interactions used in the simulations we can get accurate predictions for the functions $\Phi\left(\xi_{\text {IS }}\right)$ and $\gamma_{\mathrm{LV}}(q)+\Delta \gamma_{w}\left(q, \xi_{\text {IS }}\right)$, which define the Hamiltonian model for RG analysis, and we may check their dependence with the specific definition of the intrinsic function. Since the theoretical approaches are developed from the limit of very thick wetting layers, proving their validity for thin films, of a few monolayers and with structured density profiles, is, on its own, an interesting result of our work.

On the other hand, if we look at the whole dependence of the CW fluctuations on the film thickness (and not just its bottom line), we observe the strong layering effect, with enhanced fluctuations when $\xi_{\text {IS }}$ goes from one layer to the next. Further studies are required to test for finite-size effects and for the differences between the results of canonical and those of grand-canonical simulations for $\Phi\left(\xi_{I S}\right)$. In the simplest scenario, the oscillatory behavior of the $\mathrm{CW}$ damping could be produced by a pseudo phase transition induced by the finitesize effects. Otherwise, if such layering had to be incorporated into an effective Hamiltonian $\mathcal{H}[\xi]$, it would imply the use of nonlocal dependence with oscillatory kernels, which have not been considered in any RG analysis. The appealing consistency between $B^{\text {up }}(q)$ in Fig. 3(b) and the theoretical framework suggests that the enhanced (or subdamped) $\mathrm{CW}$ fluctuations that create the oscillatory behavior of $\Delta \gamma\left(q, \xi_{\text {IS }}\right)$ could be spurious or irrelevant in the RG flux. The present procedure may also be applied to the study of wetting layers on structured substrates where nonlocal effects could be more relevant to contrast with the predictions of Fisher and $\mathrm{Jin}^{3}$ and Parry et $a^{4,5}$ Work addressing these questions is in progress and will be reported in the future.

\section{ACKNOWLEDGMENTS}

We acknowledge the support of the Spanish Minister of Science and Innovation (Grant No. FIS2010-22047-C05) and the Comunidad Autónoma de Madrid under program MODELICO (Grant No. S2009/ESP-1691).

\footnotetext{
*efernand@icmm.csic.es

†echacon@icmm.csic.es

†pedro.tarazona@uam.es

${ }^{1}$ D. Bonn, J. Eggers, J. Indekeu, J. Meunier, and E. Rolley, Rev. Mod. Phys. 81, 739 (2009).

${ }^{2}$ S. Dietrich, in Phase Transition and Critical Phenomena, edited by C. Domb and J. Lebowitz (Academic Press, New York, 1988), Vol. 12, pp. 1-218.

${ }^{3}$ M. E. Fisher and A. J. Jin, Phys. Rev. Lett. 69, 792 (1992).

${ }^{4}$ A. O. Parry, J. Phys.: Condens. Matter 18, 6433 (1996).

${ }^{5}$ A. O. Parry, C. Rascón, N. Bernardino, and J. M. Romero-Enrique, Phys. Rev. Lett. 100, 136105 (2008).

${ }^{6}$ A. O. Parry and C. Rascón, J. Phys.: Condens. Matter 23, 015004 (2011).

${ }^{7}$ I. M. Tidswell, T. A. Rabedeau, P. S. Pershan, and S. D. Kosowsky, Phys. Rev. Lett. 66, 2108 (1991).

${ }^{8}$ L. Pang, D. P. Landau, and K. Binder, Phys. Rev. Lett. 106, 236102 (2011).

${ }^{9}$ R. Evans, Adv. Phys. 28, 143 (1979).

${ }^{10}$ P. Tarazona and E. Chacón, Phys. Rev. B 70, 235407 (2004).

${ }^{11}$ F. H. Stillinger, J. Chem. Phys. 76, 1087 (1982).

${ }^{12}$ J. Chowdhary and B. M. Ladanyi, J. Phys. Chem. B 110, 15442 (2006).

${ }^{13}$ L. Partay, G. Hantal, P. Jedlovszky, A. Vincze, and G. Horvai, J. Comput. Chem. 29, 945 (2008).
}

${ }^{14}$ M. Jorge and M. Cordeiro, J. Phys. Chem. C 111, 17612 (2008).

${ }^{15}$ D. Zhukhovitskii, J. Chem. Phys. 125, 234701 (2007).

${ }^{16}$ E. Chacón, E. M. Fernández, D. Duque, R. Delgado-Buscalioni, and P. Tarazona, Phys. Rev. B 80, 195403 (2009).

${ }^{17}$ E. Chacón and P. Tarazona, Phys. Rev. Lett. 91, 166103 (2003).

${ }^{18}$ M. Jorge, P. Jedlovszky, and N. Cordeiro, J. Phys. Chem. C 114, 11169 (2010).

${ }^{19}$ M. Jorge, G. Hantal, P. Jedlovszky, and N. Cordeiro, J. Phys. Chem. C 114, 18656 (2010).

${ }^{20}$ E. Chacón and P. Tarazona, J. Phys.: Condens. Matter 117, S3493 (2005).

${ }^{21}$ E. M. Fernández, E. Chacón, and P. Tarazona, Phys. Rev. B 84, 205435 (2011)

${ }^{22}$ E. Velasco and P. Tarazona, J. Chem. Phys. 91, 7916 (1989).

${ }^{23}$ E. Velasco and P. Tarazona, Surf. Sci. 251, 628 (1991).

${ }^{24}$ K. Binder, D. P. Landau, and D. M. Kroll, Phys. Rev. Lett. 56, 2272 (1986).

${ }^{25}$ E. Brezin and S. Leibler, Phys. Rev. Lett. 50, 1387 (1983).

${ }^{26}$ D. S. Fisher and D. A. Huse, Phys. Rev. B 32, 247 (1985).

${ }^{27}$ A. O. Parry and R. Evans, Phys. Rev. Lett. 64, 439 (1990).

${ }^{28}$ A. O. Parry and R. Evans, Physica A 181, 250 (1990).

${ }^{29}$ T. Kerle, J. Klein, and K. Binder, Eur. Phys. J. B 7, 401 (1999).

${ }^{30}$ R. Checa, E. Chacón, and P. Tarazona, Phys. Rev. E 70, 061601 (2004).

${ }^{31}$ K. R. Mecke and S. Dietrich, Phys. Rev. E 59, 6766 (1999). 
${ }^{32}$ P. Tarazona, E. Chacón, and F. Bresme, Condens. Matter Phys. 24, 284123 (2012).

${ }^{33}$ S. Dietrich and M. Napiórkowski, Phys. Rev. A 43, 1861 (1991).

${ }^{34}$ A. O. Parry, J. M. Romero-Enrique, and A. Lazarides, Phys. Rev. Lett. 93, 086104 (2004).

${ }^{35}$ E. M. Blokhuis, J. Chem. Phys. 130, 014706 (2009).
${ }^{36}$ F. B. Usabiaga and D. Duque, Phys. Rev. E 79, 046709 (2009).

${ }^{37}$ S. Pandit, D. Bostick, and M. Berkowitz, J. Chem. Phys. 119, 2199 (2003).

${ }^{38}$ R. L. C. Vink, J. Horbach, and K. Binder, J. Chem. Phys. 122, 134905 (2005). 